\title{
Micro-Raman Study of the Fatigue and Fracture Behaviour of single PA66 Fibres. Comparison with single PETand PP Fibres
}

\author{
Ph. Colomban ${ }^{1 *}$, J.M. Herrera Ramirez ${ }^{1,2}$, R. Paquin ${ }^{1}$, A. Marcellan ${ }^{1,2}$, A. Bunsell ${ }^{2}$ \\ ${ }^{1}$ Laboratoire de Dynamique, Interaction et R\&activité (Ladir), \\ UMR 7075 CNRS \& Université Pierre et Marie Curie, France; \\ ${ }^{2}$ ENS Mines de Paris, Centre des Matériaux P.-M. Fourt, UMR 7633 CNRS \\ BP 87, 91003 EVRY Cédex France \\ * corresponding author \\ fax 33149781118 \\ philippe.colomban@glvt-cnrs.fr
}

\begin{abstract}
The micro/nano structural evolution before and after tensile loading, fatigue and ultimately, failure has been studied by Raman microspectroscopy for PA66, PP, PBO and PET single fibres and precursors, using two probes : low wavenumber collective modes at $<150 \mathrm{~cm}^{-1}$ as representative of the crystalline and amorphous chains and stretching and bending modes, as representative of the $\mathrm{C}-\mathrm{C}$ bond local behaviour. Wavenumber and bandwidth distribution across fibre/precursors diameters revealed different types of skin/core heterogeneity. The in situ analysis at different strain levels shows that amorphous chains of fibre accommodate the stress differently. Slight tensile straining is observed for crystalline PA 66 chains, and compressive one for PP and PET chains indicating different local chain architectures. Post mortem analyses of a series of fibres failed in fatigue tests show that amorphous domains are highly stressed during the failure and a remnant stress can be measured.
\end{abstract}

Keywords : Raman, fibre, nanostructure, mechanics, fatigue, tension 


\section{INTRODUCTION}

Polyamides (PA) and polyethylene terephthalate (PET) are complex engineering polymers used for a wide range of industrial applications in bulk and fibrous forms [1]. In such roles the material has to exhibit high resistance to crack initiation, which imparts high toughness to un-notched materials [2,3]. Many studies have been carried out on PA and PET in order to identify the relationship between microstructure and macroscopic mechanical properties [3-7]. Theses fibres are used in the moorings of ships, ropes, straps, belting and in tyre reinforcement. In these applications the fibres are subjected to severe loadings which can induce a fall of mechanical properties, associated with a phenomenon of fatigue. Fatigue failure in thermoplastic fibres was identified more than thirty years ago [8] but the micro/nanostructural processes governing such behaviour remain the source of speculation. It is known that thermoplastic fibres such as PA 66 and PET fail by similar fatigue processes involving distinct stages of crack growth leading to distinctive fracture morphologies.

The present study examines and compares the behaviour of PA 66 (previously published in detail [9-11]) and PET fibres under fatigue loading until fracture and the correlation between the fibre (nano)structure and structural heterogeneity, with fatigue lifetimes. Comparison will be extend to two very different fibres, PP (poly(propylene)) a viscoelastic material and PBO (poly(p-phenylenebenzobisoxazole), an elastic high modulus material [7]. Emphasis is given to the comparison across the fibre of the structural differences between the fibre core and surrounding skin and to understanding the transformations induced by "cold" drawing of a "precursor" (large diameter fibres taken just after the spinneret before final drawing). Single fibres were mounted in the grips and were loaded in a Universal Fibre Tester [12], and the fibres were submitted to different strain levels up to their fracture and their Raman spectra in situ recorded. In addition, some fibres, which had been broken during fatigue tests, have been analysed at points ahead of the fatigue crack tip. Studies in which Raman microspectroscopy has been applied to single fibres are, however, relatively few [13], especially those with a spatial resolution able to compare the fibre skin and core characteristics. None of these works discuss the relationship between the polymer nanostructure and the failure behaviour.

\section{EXPERIMENTAL}

Polyamide 66 (the diameter of single fibres was about $30 \mu \mathrm{m}$ ), Poly(ethyleneterephthalate) (diameter $\sim 20 \mu \mathrm{m}$ ) and its precursor (diameter $\sim 350 \mu \mathrm{m}$ ) and poly(propylene) (diameter $\sim 50 \mu \mathrm{m}$ ) and corresponding precursor (same diameter) were studied.

Raman vibrational analysis was performed on single fibres extracted from the yarn as described in refs $[10,11]$. These previous studies demonstrated that the size applied to the fibre surface during manufacture does not contribute to the Raman spectra in the analysed wavenumber ranges.

A "XY" spectrographs (Dilor, Lille, France) equipped with a double monochromator filter and a back-illuminated, liquid nitrogen-cooled, 2000 x 256 pixels CCD detector (Spex, Jobin-Yvon-Horiba Company) was used to record Raman spectra between 10 and 2000 $\mathrm{cm}^{-1}$, using 514.5 (PA 66, PET, I-PP) and $647.1 \mathrm{~nm}$ (PBO) exciting lines ( $\mathrm{Ar}^{+}-\mathrm{Kr}^{+}$laser). The power of illumination ranged between $1 \mathrm{~mW}$ and $10 \mathrm{~mW}$. Backscattering illumination and collection of the scattered light were made through an Olympus confocal microscope (long focus Olympus x50, x80 or x100 objective, total magnification x500, x800 or x1000). 
Although PET and PA66 fibres show rather similar mechanical behaviours with a 3-regime stress-strain plot (Fig.1), the PP fibre curve exhibits a 2-regime behaviour with a very large viscoelastic plateau. The fibres also have different densities (i.e. number of chemical bond per volume unit). The rather large strains obtained with some of these fibres under imposed strain conditions has required the use of a Universal Fibre Tester described in refs [10-12] with a fixed grip and a mobile grip (Fig. 2a). A single fibre was first stuck on a paper frame using neoprene glue (Fig. 2a) which was cut before the experiment. The laser was focused through the microscope objective and the same objective used to collect the scattering light. The different strain levels were applied on single fibres at a loading strain rate of $100 \% \cdot \mathrm{min}^{-1}$, i.e. a speed of the mobile grip of $1.6 \times 10^{-2} \mathrm{~s}^{-1}$. After stabilisation of the viscoelastic behaviour (about 5 minutes), i.e. stabilisation of stress versus time, Raman spectra were recorded from $t_{a c q=0}$ to $t_{\text {end }}$ according to the procedure described in ref. [10]. The recording time ranged between $\sim 600$ (PET) and $3000 \mathrm{~s}$ (PA). The skin/core substructure was studied by linear scanning measurements (1D cartography) across the fibre diameter. The laser was focused on the fibre edges (spot size $\sim 5 \mu \mathrm{m}^{2}$, with a $\mathrm{x} 100$ objective, i.e. x1000 magnification) and was polarised either parallel to the fibre axis (noted //) or perpendicular (noted $\perp$ ) by changing the alignment of the fibre with respect to the spectrometer slit [11]. Consequently, although the stress/strain curve took a few tens of seconds to obtain, a Raman study of a fibre loaded at different levels up its fracture took several hours. At least 3 fibres are analysed for each type of fibre and measurements were made at several places along each specimen.

Fibre precursors (Fig. 2b) were mounted on a second Universal Fibre Tester designed for larger strain values. Some drawing was also performed by hand.

Fibres can be considered as being similar to a uniaxial crystal with $C_{\alpha}$ symetry. As for any crystal, the different components of the polarisation tensor are obtained from measurement of the Raman spectra according to the different combinations of the crystal orientation vs the horizontal/vertical polarised laser beam and the direction of the measured scattered light [14]. The number of measurements varied as a function of the crystal symmetry, , e.g. from 6 for low symmetry to 2 for high symmetry $C \alpha$ [15]. Note, the quality of the polarisation measurement is less good when measurements are made through a microscope and errors in intensity measurements arise from polarization scrambling due to reflection and refraction [14]. Thus we will only consider the bigger changes. Qualitative measurements of the orientational distribution function in poorly crystallized fibres (leading to poor polarised spectra) have been proposed, using the coefficients of Legendre polymonials, by different authors (see ref. [13] for a review) and recently used for silk fibres [15].

The baseline was first removed with Labspec ${ }^{\mathrm{TM}}$ (Dilor, France) and the spectra were decomposed with the ORIGIN ${ }^{\top M}$ software (MICROCAL ${ }^{\mathrm{TM}}$ Software, Inc., USA) as described in previous papers $[11,12,16,17]$. The following assumptions are made : a single Raleigh line, Lorentzian shape for narrow peaks and a Gaussian form for broad peaks The Raman fingerprint of air (series of narrow peaks near $60 \mathrm{~cm}^{-1}$ ) is used as an internal standard and the fitting of these peaks is mandatory to describe precisely the Rayleigh wings. The intensity of the air signature is related to the number of bubbles or cracks in the analysed volume.

\section{RESULTS}




\section{Choice of the Raman probes}

In the past, most Raman studies have concerned only the middle wavenumber range, the bending domain and stretching modes [18-23]. Information obtained from these modes is very local. High resolution instruments now make it possible to analyse the low wavenumber region, where modes have a collective character. This is obvious from the comparison of polarized spectra (Figs 3 and refs. [10,11]): the modes at about $80-100 \mathrm{~cm}^{-1}$ assigned to a lattice mode involving the translational/librational movement ( $T$ ') of the chains of the unit-cell exhibit the stronger degree of polarization. Roughly speaking, this mode can be described as a translation of the whole chain with respect to adjacent ones. This mode involves modifications of the electron-richer bonds and hence gives rise to very strong peaks. The greater the number of atoms involved in the motion, the lower the wave number will be. On the other hand, inter-chain coupling will shift the peak toward high wavenumbers. These low wavenumber collective modes may be preferred for the study of the relationship between the chain structure and the macroscopic mechanical behaviour. On the other hand, the assignments of bending and stretching modes are well documented, especially their relationship with conformational changes [18-31]. Note, local modes such as $\mathrm{N}-\mathrm{H}$ stretching mode can also a good source of information about the modification of neighbouring chains arrangements [9-11,16,17].

In Raman spectroscopy, the probe is the chemical bond itself (through the bond polarizability). Thus, as a function of the vibrational mode concerned, the analysis will be more or less sensitive to the local order/disorder, very local $(0.2-0.5 \mathrm{~nm})$ for stretching modes, larger for bending modes which reflect the disorder of neighbouring entities (0.5-1 $\mathrm{nm}$ ) and largest for lattice/librational modes involving the motion of the whole vibrational unit $(0.5-10 \mathrm{~nm})$. This has a great importance in the understanding of the Raman signature of polymers. Fig. 5 shows a schematic view, in which chains are organized in different geometries. At the very local scale we can considered two types of chain: those with adjacent ordered chains (in "crystallites" but also in regions inside the circles, the so-called “oriented amorphous” region of X-ray studies [9]) and chains with disordered neighbouring chains. In polymeric structures orientational bond disorder is less energetic than translational ones and it is rather difficult to discriminate between biphasic material (made of crystallites and of amorphous domains) and Hosemann' paracrystals (continuous orientational disorder, without origin or distinct borders ) [1,11,32]. Both cases lead to similar correlation lengths in X-ray diffuse scattering. Although the cut-off threshold will be different as a function of the vibrational probe (stretching, bending or collective modes), some data could allow the two above types of disorder to be discriminated.

\section{Fibre drawing and axial anisotropy of the chain arrangement}

Fig. 3 compares the Raman signatures of pristine PET precursors (Fig. 2b), (hand)stretched precursors and standard single fibres. Strong modifications of the low wavenumber domains are obvious, with the appearance of two polarised peaks at $\sim 73$ and $129 \mathrm{~cm}^{-1}$, the disappearance of the broad component below the $\sim 860 \mathrm{~cm}^{-1}$ peak and the creation of the 995, 1097 and $1185 \mathrm{~cm}^{-1}$ peaks (Fig. 6). All these modes involve the ring deformation and those with rising intensity have been assigned to crystalline, trans PET [25-31,33,34], although the $\sim 1175 \mathrm{~cm}^{-1}$ peak of the precursor has been associated to "amorphous" material. It is thus evident that drawing develops the trans conformation and increases both the crystallinity and the collective orientation of the chains: a plot of the relative $995 \mathrm{~cm}^{-1}$ peak area shows a drastic increase from 0.2 to 0.65 after $100 \%$ strain and then a slower rate 
up to 0.8 for $700 \%$ strain. The $898 \mathrm{~cm}^{-1}$ peak assigned to gauche conformation decreases accordingly. Note that during the hand drawing and diameter reduction (Fig. 2b) the fibre temperature increased to $\sim 60-80^{\circ} \mathrm{C}$. Thus it is clear that the local temperature can be much higher in some locations, as a function of local structure, strain rate and heat transfer than the surrounding environment. Note also that the spectral changes seem more related to the drawing rate than to achieved strain. Comparison of the standard and extended fibre (see for instance the spectrum of the single PET fibre stretched at 630MPa in Fig. 6) shows that the degree of trans conformation decreases when the fibre is highly stressed, just before its fracture.

Rather similar behaviour is observed for the PP precursor. Although polarization of precursor spectra is very poor, spectra of $500 \%$ stretched single fibre show a highly polarized fibre, according to a high orientation of the PP chain along the fibre axis. Nielsen et al. and Arrueberra de Baez [35-36] have assigned the $\sim 810\left[\mathrm{r}_{\mathrm{CH} 2}+v_{\text {C-C }}\right]$ and $\left[\mathrm{r}_{\mathrm{CH} 2}\right] \sim 840$ $\mathrm{cm}^{-1}$ peaks to crystalline long and shorter helical chains, respectively. Thus a measure of the crystallinity/axial orientation of the chains can be made from the area ratio of these two bands for the two different polarization: $\mathrm{n}_{1}=\left[\mathrm{A}_{841} / \mathrm{A}_{808}\right]_{/ /} /\left[\mathrm{A}_{841} / \mathrm{A}_{808}\right] \perp=1.5$ in the precursor to 1.7 in the x $500 \%$ stretched single fibre). However the low wavenumber signature leads to a more representative measurement of the long range ordering : $\mathrm{n}_{1}$ $=\left[\mathrm{A}_{100} / \mathrm{A}_{400}\right]_{/ /} /\left[\mathrm{A}_{100} / \mathrm{A}_{400}\right] \perp=0.9$ in the precursor and 3.65 in the $\mathrm{x} 500 \%$ stretched fibre. As a first conclusion, different tools are available to characterize the crystallinity and the chain orientation in the fibre section and along the fibre axis.

\section{Skin/core heterogeneity}

Because of its micronic spatial resolution, Raman microspectroscopy is a good technique to analyse specifically all kinds of single fibre sections [37]. The existence of structure and a symmetric residual stress gradient in the fibre section was demonstrated for the first time using micro-Raman spectroscopy in PA 66 single fibre and provides an explanation of fracture initiation mechanism [10,11]. Recently synchrotron radiation appears as an alternative (but more expensive) method to obtain similar results in hair [38] and PBO single fibres [39]. Figs 7 and 8 show representative plots of the low wavenumber crystalline and amorphous components recorded along line scan across a fibre diameter for PA 66 and PBO single fibres and for PET precursors and single fibres.

As shown in Fig. 4, the low wavenumber Raman signature of "crystalline" chains is obvious, even for a material scientist without expertise in spectroscopy. The Raman signature of the crystalline material, and of non-distorted molecules, is a Lorentzian curve. The shape of the Rayleigh line wing can also be described using a Lorantzian. On the other hand, in disordered or glassy materials, in which there is a distribution of configuration, the envelope of all the Raman signature is usually described with a Gaussian curve. More complex band shapes are possible. We postulate that the signature of amorphous chains, homologous to the crystalline ones, will be present at lower wavenumbers, because the lower density of amorphous matter involves larger interchain distances and weaker energy for $T$ ' modes. In order to achieve a good description of the Raman spectrum we need to incorporate, in the fit, the signature of air (very narrow peak series with bandwidth determined by the instrument resolution, see for instance the I-PP spectrum in Fig. 4). With this fitting procedure we can separate the contribution of amorphous and crystalline chains $[11,17]$. A similar procedure can be applied to N-H stretching modes after isotopic dilution in deuterated materials [17]. 
Largest variations are always observed in the signatures of amorphous chains but they differ with each type of fibre, and for the same material as a function of the mechanical (precursor/fibre stretching degree) or thermal treatment: for instance we observe a shift up of the wavenumber for the amorphous PA 66 chain in the fibre core from 65-70 to 95 $\mathrm{cm}^{-1}$ (Fig. 7a), toward the position of the crystalline chains $\left(v=100 \mathrm{~cm}^{-1}\right)$. Accordingly, the FWHM decreases from $\sim 65 \mathrm{~cm}^{-1}$ in the fibre skin to about $35 \mathrm{~cm}^{-1}$ in the fibre centre: the crystallinity is a maximum at the centre of the PA 66 fibre section [11]. The heterogeneity disappears after a thermal annealing above Tg [11,40]. On the contrary a shift down is observed for PET fibre from the periphery to the fibre centre (Fig. 7b). The bandwidths of both amorphous and crystalline signatures (Fig. 8) remain constant indicating that the wavenumber shift is due to a tensile residual stress of the amorphous chains in the core. PBO fibre section shows rather similar behaviour to that of PA 66 fibre: the bandwidth of the amorphous chain signature (described by the mean of a broad Rayleigh wing because the contribution cannot be separated from the Rayleigh peak) strongly increases in the fibre centre. This is consistent with texturing investigations made with X-ray scattering and micro-focus synchrotron beam [39,41] which demonstrate that the rigid ribbon-like PBO chains are parallel to the fibre axis and form a radial fibrillar texture. Disorder is a maximum at the fibre centre.

The line-scan cartography across fibre diameter provides an evaluation of the domain sizes to be assessed and the residual stress state $[10,11]$.

\section{Fibre extensometry - The relationship between nanomechanics and nanostructure}

On the basis of chemical bonds, anharmonicity, any-stress induced interatomic distance alteration should change the atomic vibration wavenumbers. This is the principle of Raman extensometry [42,43]. The empirical relationship between the strain-induced Raman wavenumber sfhift $(\Delta v)$ to tensile strain $(\Delta \varepsilon)$ is linear [41-49]:

$$
\Delta v=\mathrm{S}^{\varepsilon} \mathrm{x} \Delta \varepsilon
$$

and the above equation is microscopically analogous to Hooke's law

$$
\Delta \sigma=\mathrm{E} \times \Delta \varepsilon
$$

and

$$
\Delta v=S^{\varepsilon} \times(100 \times \Delta \sigma) / E=S^{\sigma} \times \Delta \sigma
$$

Young's modulus is indeed the result, at the macroscopic scale, of the force constant of the various chemical bonds. Consequently, it has been established $[48,49]$ that

$\mathrm{S}^{\varepsilon}=\mathrm{k}^{1} \mathrm{E}^{-1 / 2}$

Following on from work previously reported for PA 66 fibres, the variation due to straining of the amorphous and crystalline chain low wavenumber signatures of PET and i-PP fibres was studied and compared. Very different behaviours are observed for crystalline and amorphous signatures. As for the stress/strain curve recorded using the Universal Testing Tester two or more important stages in the mechanisms of deformation can be seen in Fig. 9. The initial plateau behaviour, observed for both crystalline and amorphous chains signature, is consistent with an initial alignment of the chains without energy dissipation up to a threshold which differs as a function of the molecular chain (PP $50 \mathrm{MPa}$ (5\% of strain), PET $300 \mathrm{MPa}(4 \%)$ and PA $66 \sim 300-550 \mathrm{MPa}(10-12 \%)$ ) and also as a function of the fibre grade and producer (e.g. difference between PA 66-A and PA 66-B fibres [11]). Marked differences also exist between amorphous and crystalline chains. Above this threshold two opposite behaviours are observed for the signature of crystalline chains : i) a very small increase in wavenumber is observed for PP and PET (followed by a plateau regime), indicating that the fibre stretch leads to a slight compression of the crystalline chains; ii) a small down-shift is observed for PA 66 fibre. These two opposite behaviours 
provide information about the structural arrangement, in particular the discrimination between biphasic and paracrystal models (see further).

The downshift rates measured for amorphous chain signatures are given in Table 1 . The larger reactivity to the stress of the amorphous chains results from much larger $\mathrm{S}$ values. It is obvious that the $S^{\varepsilon}$ absolute value increases as the density and the glass transition temperature:

$$
\begin{aligned}
& T g_{i-P P}<T g_{P A-66}<T g_{P E T} \\
& d_{i-P P}<d_{P A-66}<d_{P E T}<d_{P B O} \\
& S^{\varepsilon}{ }_{i-P P}<S^{\varepsilon}{ }_{P A-66}<S^{\varepsilon}{ }_{P E T}
\end{aligned}
$$

This rather large difference between $S$ values highlights the difference in stiffness - and structure - of the amorphous chains/regions of PP and other fibres.

The comparison of the macroscopic (Stress/strain plot) and nanometric (wavenumber shift versus applied strain) shows that stage threshold observed at the chain scale and at the macroscopic scale almost coincide : up to the stage 2-stage 2' transition, we observe a compression by matrix Poisson' effect of PET crystallites, then in stage 2', the amorphous chains are stressed gently and in stage 3 drastically up to the fibre fracture. We believe that the long duration of a Raman measurement makes it not possible to explore all the stage 3 because the fibre breaks earlier in the plateau of regime 3.

A chain arrangement such as that proposed by Oudet [2,5] supposes that crystalline and amorphous chains are distributed in separate regions. Such an arrangement is consistent with the PET and i-PP behaviours where a up-shift or plateau is observed for crystalline chains although a down-shift is measured for amorphous ones. On the contrary, the PA 66 behaviour where crystalline and amorphous chains follow similar behaviours, but at different rates, is more consistent with a series arrangement as proposed by paracrystal [32,50] and Prevorsek's models and is consistent with the previous conclusions based on isotopic dilution studies [17].

\section{Origin of the fatigue}

In Fig. 10a we compare the wavenumber shift measured for amorphous PA66 chains as a function of the applied strain (the example shown was obtained with fibre A [11]) and measured at the fatigue fracture point (point B): it can be seen that the data measured at point $\mathrm{B}$ corresponded to the expected shift value if the accommodation due to the energyfree movement of the chain were suppressed. Fig. 10b shows the results from the measurements made close to the zone of rupture after fatigue. It can be seen that the differences in residual stress were very similar at points A and C. In contrast, large differences were observed at point B compared with the values measured in other places. This reveals that the amorphous chains have been compressed during the fatigue fracture (this behaviour is not observed for fibre cut with a razor blade or broken in tensile test [11]) and that part of the stress is residual. During the fatigue, the amorphous chains/regions in the fibre lose compliance so that the covalent bonds in the main chains are repeatedly stressed during cyclic load. Observations of the fracture seem to indicate local heating according to the exothermic precursor stretching. The origin of this large remnant compressive stress can be found in the fast cooling taking place with the fibre fracture. 


\section{CONCLUSION}

The micro/nano mechanisms of deformation and the property gradient, between surface and fibre core, have been investigated using low wavenumber Raman collective modes $(\sim 100$ $\left.\mathrm{cm}^{-1}\right)$. The main roles played by amorphous domains and crystallites have been revealed and show the important contribution of the amorphous regions. This skin/core effect seems to vary with fibre type and grade/producer, because of the different thermomechanical steps during the manufacturing process. It is obvious that the first microns of the fibre skin experience different strains, temperature and cooling rate, etc. from the core of the fibre. Viscosity differences of short-chains and long-chain polymers will also affect their distribution in the fibre section at the spinneret exit. A residual compression stresses in skin can be evaluated using the relationship between wavenumber shift and applied strain $\left(S^{\sigma}\right)$. Because of the different thermo-mechanical steps during the process the first tens of microns of the fibre experience different strains from the core of the fibre.

The different stages observed on macroscopic stress/strain curves have been related to the different stages observed on the Raman extensometry plots. The latter behaviour could reflect the transition of the micro-mechanisms of deformation from a progressive alignment of amorphous macromolecules to an increase of nano-heterogeneities due to kink formation and stresses in entanglements. Differences or similarity in the behaviour of amorphous and crystalline chains have been used to assess the best schematic for the chain arrangements, This suggest that in polyamide fibres, where both components undergo down shift with stress, the crystalline, oriented amorphous and random amorphous phases are primarily in series (paracrystal model) although for PP and PET fibre a biphasic model fit well the differences in the behaviour of crystalline and amorphous components.

Analyses of the stress states ahead of the fatigue crack tip of PA 66 fibres have also confirmed that the effect of fatigue loading on the fibre structure extended a considerable way ahead of the crack. It has been shown that, during fatigue, the amorphous regions in the fibres lose compliance so that the covalent bonds in the main chains in the amorphous regions are repeatedly stressed during cyclic loading.

\section{ACKNOWLEGMENTS}

Gérard Sagon is acknowledged for his technical help in Raman spectra recording and C. Le

Clère for the strain/stress curve measurements. 


\section{REFERENCES}

1. Walczak ZK., Processes of Fiber Formation, Elsevier, 2002, Amsterdam.

2. Oudet C. , Polymères, structure et propriétés, Masson, Paris, 1994.

3. Bunsell AR., Reinforcing fibers in Encyclopedia of Applied Physics, VCH Publishers, 1996, pp 343-368.

4. Prevorsek DC,Harget PJ, Sharma RK, Nylon6 fibers : change in structure between moderate and high draw ratios, J. Macromol. Sci. B 1973; 8: 127-156..

5. Oudet Ch, Bunsell AR, Effect of structure on the tensile, creep and fatigue properties of polyester fibres, J. Mater. Sci. 1987; 22: 4292-4298.

6. Suzuki A., Nakamura Y., Kunugi T., Microstructure and mechanical properties of Hot-air drawn poly(ethylene terephthalate) fibers, J. Polymer Sci. Part B Polymer Phys. 199; 37; 1703-1713.

7. Kitagawa T., Yabuki K., Young R.J., An investigation onto the relationship between processing, structure and properties for high-modulus PBO fibres. Part 1. Raman band shifts and broadening in tension and compression, Polymer 2001; 42: 21012112.

8. Bunsell AR, Hearle JWS, A mechanism of fatigue failure in nylon fibres, J. Mater. Sci. 1971; 6: 1303-1311.

9. Marcellan A, Bunsell AR, Piques R, Colomban Ph, Micromechanisms, mechanical behaviour and probalistic fracture analysis of PA 66 fibres, J. Mater. Sci. 2003; 38: 2117-2123.

10. Marcellan A, Colomban Ph, Bunsell AR, (Nano)structure, skin/core and tension behaviour of polyamide fibres, J. Raman Spectrosc. 2004; 35:308-315 .

11. Herrera Ramirez J.M., Colomban Ph., Bunsell A.R., Micro-Raman study of the fatigue fracture and tensile behaviour of polyamide (PA 66) fibres, J. Raman Spectrosc. 2004; 35: 1063-1072.

12. Bunsell A.R., Hearle J.W.S., Hunter R.D., An apparatus for fatigue-testing of fibres, J. Phys. E-Scientific Instruments, 1971; 4: 868-872.

13. Dhamelincourt P., Nakashima SI., Application to material science, ch. 6, in Raman Microscopy, Developments and Applications, Turrell G. \& Corset J., Eds, Academic Press, London, 1996, pp 280-285.

14. Poulet H, Mathieu J-P, Spectres de vibration et symétrie des cristaux, Gordon \& Breach, Paris, 1970.

15. Rousseau ME, Lefèvre T, Beaulieu L, Asakura T, Pezolet M, Study of protein conformation and orientation in silkworm and spider silk fibers using Raman microspectrometry, Biomacromolecules 2004; 5: 2247-2257.

16. Colomban Ph., Sagon G., Lesage M., Herrera Ramirez J.M., Micro-Raman study of the compressive behaviour of advanced PA 66 polyamide fibres in a diamond-anvil cell, Vibrational Spectroscopy, 2005; 37: 83-90.

17. Colomban Ph., Micro-analyse Raman et IR de fibres à haute performance en traction et compression, in Matériaux Composites, Comptes-rendus des 14èmes Journées Nationales sur les Composites (JNC 14), Compiègne, 22-24 mars 2005, vol.1, M.L. Benzeggagh, J. Lamon Eds, AMAC, Paris, pp 3-12 (ISBN 2-95051176-7).

18. Penel-Pierron L, Depecker C, Séguéla R, Lefevre J-M, Structural and Mechanical Behavior of Nylon 6 Films Part I. Identification and Stability of the crystalline Phases, J. Polymer Sci.: Part B: Polymer Physics 2001; 39: 484-495. 
19. Ferreiro V., Depecker Chr., Laureyns, Coulon G., Structures and morphologies of cast and plastically strained polyamide 6 films as evidenced by confocal Raman microspectroscopy and atomic force microscopy, Polymer 2004; 45: 6013-6026.

20. Hendra PJ, Maddams WF, Royaud IAM, Willis HA, Zichy V, The application of Fourier transform raman spectroscopy to the identification and characterisation of polyamides, I, single number nylons, Spectrochimica Acta Part A: Mol. Spectr. 1990; 46: 747-756.

21. Vasanthan N, Salem DR, Infrared spectroscopic characterization of oriented polyamide 66 : band assignment and crystallinity measurement, J. Polymer Sci.: Part B: Polymer Physics 2001; 38: 516-524.

22. Elzein T, Brogly M, Schultz, Crystallinity measurements of polyamides absorbed as thin films, Polymer 2002; 43: 4811-4822.

23. Vasanthan N, Salem DR, FTIR Spectroscopic Characterization of Structural change in Polyamide 6 Fibres during Annealing and Drawing, J. Polymer Sci.: Part B: Polymer Physics 2001; 39: 536-543.

24. Stuart B.H., Polymer crystallinity studied using Raman spectroscopy, Vibrational Spectrosc. 1996; 10: 79-87.

25. Purvis J., Bower D.I., Molecular Orientation in Poly(ethylene terephthalate) by Means of Laser-Raman Spectroscopy, J. Polymer Sci. Polymer Phys. Ed., 1976; 14: 1461-1484.

26. DeBlase F.J., McKelvy M.L., Lewin M., Bulkin B.J., Low-frequency Raman Spectra of Poly(ethylene terphthalate), J. Polymer Sci. Polymer Lett. Ed. 1985; 23: 109-115.

27. Bulkin B.J., Lewin M., DeBlase F.J., Conformational Change, Chain Orientation, and Crystallinity in Poly(ethylene terphthalate) Yarns: Raman Spectroscopic Study, Macromolecules 1985; 18: 2587-2594.

28. Ellis G., Roman F., Marco C., Gomez M.A., Fatou J.G., FT Raman study of orientation and crystallization processes in poly(ethylene terephthalate), Spectrochimca Acta A 1995; 51: 2139-2145.

29. Yang S., Michielsen S., Determination of the Orientation Parameters and the Raman tensor of the $998 \mathrm{~cm}-1$ Band of Poly(ethylene terephthalate), Macromolecules 2002; 35: 10108-10113.

30. Lesko CCC., Rabolt J.F., Ikeda R.M., Chase B., Kennedy A., Experimental determination of the fiber orientation parameters and the Raman tensor of the 1614 $\mathrm{cm}^{-1}$ band of poly(ethylene terphthalate), J. Molecular Struct. 2000; 521: 127-136.

31. Maddams W.F., Royaud I.A.M., The application of Fourier transform Raman spectroscopy to the identification and characterisation of polyamides, II. Double number nylons, Spectrochimica Acta A 1991; 47: 1327-1333.

32. Guinier A., Théorie et technique de la radiocristallographie, Dunod, Paris, 1956, pp615-620.

33. Rodrigez-Cabello J.C., Quintanilla L., Pastor J.M., Fourier Transform Raman Study of the Conformer in Poly(ethylene terphthalate), J. Raman Spectrosc. 1994; 25: 335344.

34. Bahl S.K., Cornell D.D., Boerio F.J., McGraw G.E., Interpretation of the vibrational spectra of poly(ethylene terphthalate), Polymer Lett. Edition, 1974; 12: 13-19.

35. Nielsen A.S., Batchelder D.N., Pyrz R., Estimation of crystallinity of isotactic polypropylene using Raman spectroscopy, Polymer, 2002; 43: 2671-2676.

36. Arrueberra de Baez M., Hendra P.J., Judkins M., The Raman spectra of oriented isotactic polypropylene, Spectrochimca Acta 1995; 51: 2117-2124. 
37. Colomban Ph., Raman analysis and "smart" imaging of nanophase and nanosized materials, Spectroscopy Europe, 2003; 15[6]: 8-16.

38. Dumas P., Tobin M.J., A bright source for infrared microspectroscoy: synchrotron radiation, Spectroscopy Europe, 2003; 15[6]: 17-23.

39. Davies R.J., Eichhorn S.J. Riekel C., Young R.J., Crystallographic texturing in single poly(p-phenylene benzobisoaxole) fibres investigated using synchrotron radiation, Polymer 2005; 46: 1935-1942.

40. Herrera Ramirez J.M., Colomban Ph., Bunsell A., $\mu$ RAMAN Determination of Tg in Polymer Fibres: The Example of PA66 fibres, Proc. Polymer Fibres 2004, UMIST Conference Centre, Manchester, UK, 14-16 july 2004; .

41. Kitagawa T., Ishitobi M., Yabuki K., An analysis of the deformation process on poly-p-phenylenebenzobisoxazole fiber and a structural study of the newhighmodulus type PBO HM${ }^{+}$fiber, J. Polymer Sci/ Polymer Phys. 2000; 38: 1605-1611.

42. Colomban Ph., Analysis of strain and stress in ceramic, polymer and metal matrix composites by Raman spectroscopy, Adv. Engn Mater. 2002; 4: 535-542.

43. Young R.J., Yeh W.-Y., Chain stretching in a poly(ethylene terephthalate) fibre, Polymer 1994; 35: 3844-3847.

44. Young RJ, Raman Spectroscopy and mechanical Properties, in: Characterization of Solid Polymers (Ed. S.J. Spells), Chapman \& Hall, London, 1994, 224.

45. Beyerlein IJ, Amer MS, Schadler LS, Phoenix SL, New methodology for determining in situ fiber, matrix, and interface stresses in damaged multifiber composites, Sci. and Eng. Comp. Mater. Vol. 7 1998, 1-2, 151-204.

46. Yeh W.-Y., Young R.J., Deformation processes in poly(ethylene terephthalate) fibers, J. Macromolecular Sci. -Physics, 1998; 37: 83-118.

47. Yeh W.-Y., Young R.J., Molecular deformation processes in aromatic high modulus polymer fibres, Polymer, 1999; 40: 857-870., 1998; 37: 83-118.

48. Gouadec G., Colomban Ph., Non-Destructive Mechanical Characterization of SiC Fibers by Raman Spectroscopy. J. Eur. Ceram. Soc., 2001; 21:1249-1259

49. Gouadec G., Colomban Ph., Raman spectroscopy of nanomaterials, How spectra relate to disorder, paticle size and mechanical properties, Progr. Crystal Growth Character., in press.

50. Hosemann R., Crystallinity in high polymers, especially fibres, Polymer 1962; 3: 349-392. 


\section{FIGURE CAPTIONS}

Fig. 1: Stress-Strain relationships for the studied fibres (PET,PA66 and I-PP). Comparison is made with a high modulus PBO (poly(p-phenylenebenzobisoxazole) and correlation between ultimate strength and density is given.

Fig. 2 : a) Detail of the experimental setting showing the focused laser beam on the single filament fibre under controled load. b) Diameter change observed during the drawing of a PET precursor.

Fig. 3 : Polarised Raman spectra for a) pristine PET precursor (see micrograph in Fig. 2b), hand stretched precursor ( $\sim$ X 10), standard PET fibre and b) for x5 stretched single IPP fibre.

Fig. 4 : Low wavenumber parallel polarised Raman spectra for PET, PP and PA 66 fibres. Amorphous and crystalline chain components have been deduced. Note, the very narrow components are the Raman signature of air in microcracks and/or bubbles within the fibre.

Fig. 5: Schematic view of the chain arrangements according to Prevorsek [4] and Oudet $[2,5]$ where crystalline and amorphous chains are more or less organised in series (paracrystal) or in parallel (ordered domains). Ordered parallel chains (including those in the circles often called oriented amorphous phase) will be considered as crystalline chains from the Raman point of view $[9,10]$. On the other hand, other chains will be considered as amorphous.

Fig. 6 : Comparison of parallel polarised Raman spectra recorded for more or less stretched PET precursor and for standard (Std) and $630 \mathrm{MPa}$ stretched fibre with the Universal Fibre Tester. HS : hand stretched ; x 700\% : stretched x 7 with the UFT.

Fig. 7 : Plots of the crystalline (a), PA 66, 5 different fibres) and amorphous (a), PA 66 ; b) PET, 4 different fibres) component wavenumbers measured along line scans across fibre diameters for free standing fibres. PA 66 fibres are examined as-received (Std) and after different thermal treatments from 0.7 to $1.4 \mathrm{Tg}$ (after ref [11]).

Fig. 8 : Plots of a) the PET (amorphous and 75 (crystal 1) and $130 \mathrm{~cm}^{-1}$ (crystal 2) components) and b) PBO (amorphous) component full width at half maximum (i.e. the short-range disorder) measured along line scan across a fibre diameter for free standing fibres.

Fig. 9 : Wavenumber shift of the chain “ $\mathrm{T}$ ” “ mode (parallel polarisation) plotted as a function of increasing applied stress for i-PP and PA 66 fibres (strain for i-PP and PET). Crystalline and amorphous components are given and a comparison is made with macroscopic stress/strain behaviour for I-PP and PET fibres. Two series of PA 66 fibres 
from two different suppliers are analysed $:$ black squares $=$ A fibre, open squares $=\mathrm{B}$ fibre.

Fig. 10 : a) Comparison between the amorphous wavenumber shift measured as a function of the applied stress and the core maximum wavenumber shift measured at the fatigue fracture point for PA 66 fibre ; b) example of the amorphous maximum wavenumber of the " $\mathrm{T}$ '” mode measured along line scan along the fibre axis from fracture B point (0 distance) to A point $(\sim 1000 \mu \mathrm{m})$ and symmetrically; fibre tested for $25 \times 10^{6}$ cycles (after $[11]$ ). 
Table 1 : $S^{\varepsilon}$ and $S^{\sigma}$ shift rates measured for the low wavenumber amorphous chain signatures for PP, PET and PA 66 fibres.

\begin{tabular}{lll}
\hline amorphous & $\mathrm{S}^{\varepsilon} \mathrm{cm}^{-1} / \%$ & $\mathrm{~S}^{\sigma} \mathrm{cm}^{-1} / \mathrm{MPa}$ \\
\hline PP & -0.4 & -0.1 \\
PET & -4.6 & -0.04 \\
PA 66-A & -3.8 & -0.04 \\
PA 66-B & -1.1 & -0.01 \\
\hline
\end{tabular}

Table 2 : $S^{\varepsilon}$ and $S^{\sigma}$ shift rates measured for the low wavenumber crystalline chain signatures for PP, PET and PA 66 fibres.

\begin{tabular}{lll}
\hline crystalline & $\mathrm{S}^{\varepsilon} \mathrm{cm}^{-1} / \%$ & $\mathrm{~S}^{\sigma} \mathrm{cm}^{-1} / \mathrm{MPa}$ \\
\hline PP & n.a. & +0.005 \\
PET & n.a. & +0.003 \\
PA 66-A & -0.3 & -0.004 \\
PA 66-B & -0.2 & -0.025 \\
PBO [7]* & -4.85 & -0.003
\end{tabular}

* measured from the $1614 \mathrm{~cm}^{-1} \mathrm{C}$-C stretching mode n.a; : not available 


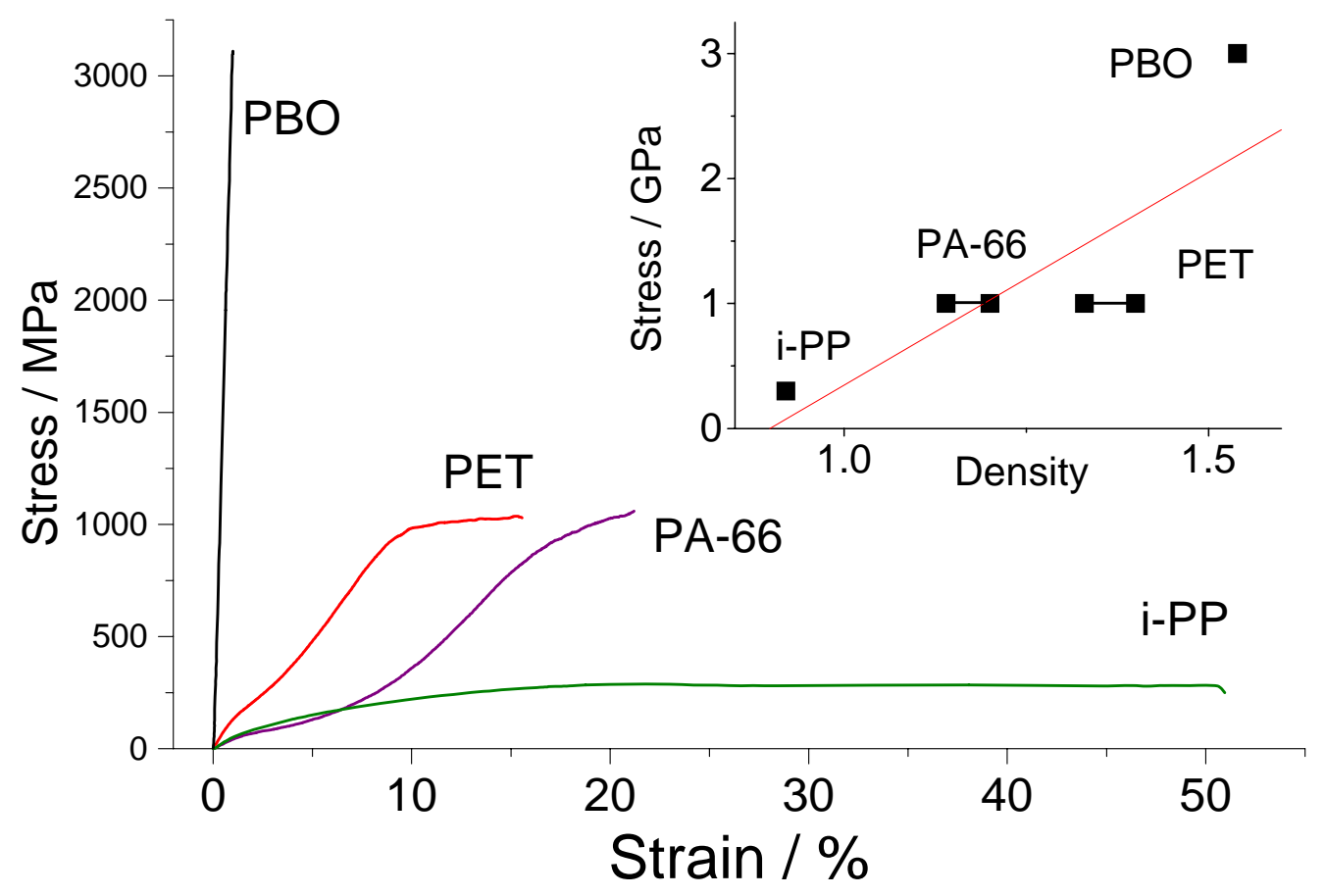

Fig. 1: Stress-Strain relationships for the studied fibres (PET,PA66 and I-PP). Comparison is made with a high modulus PBO (poly(p-phenylenebenzobisoxazole) and correlation between ultimate strength and density is given.

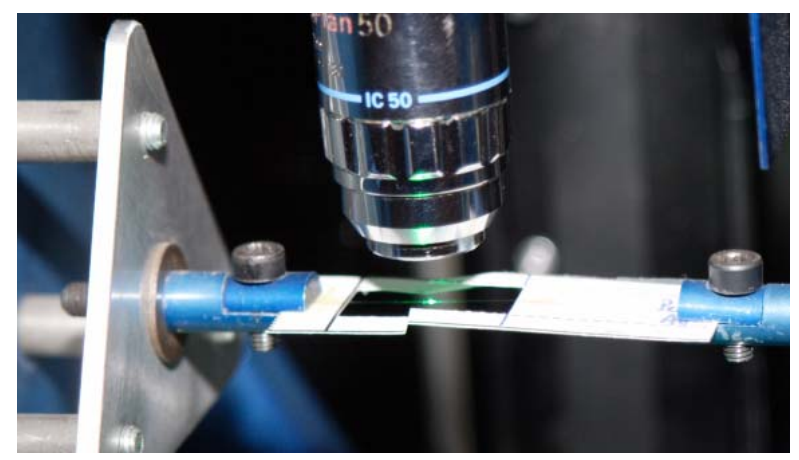

a)

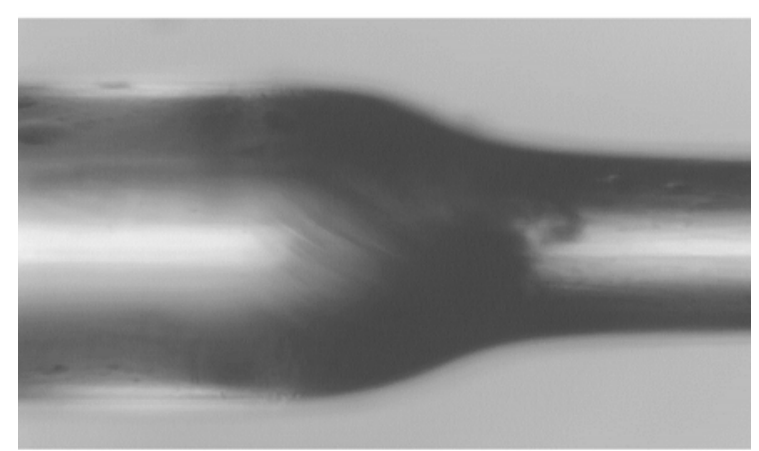

b)

Fig. 2 : a) Detail of the experimental setting showing the focused laser beam on the single filament fibre under controled load. b) Diameter change observed during the drawing of a PET precursor. 

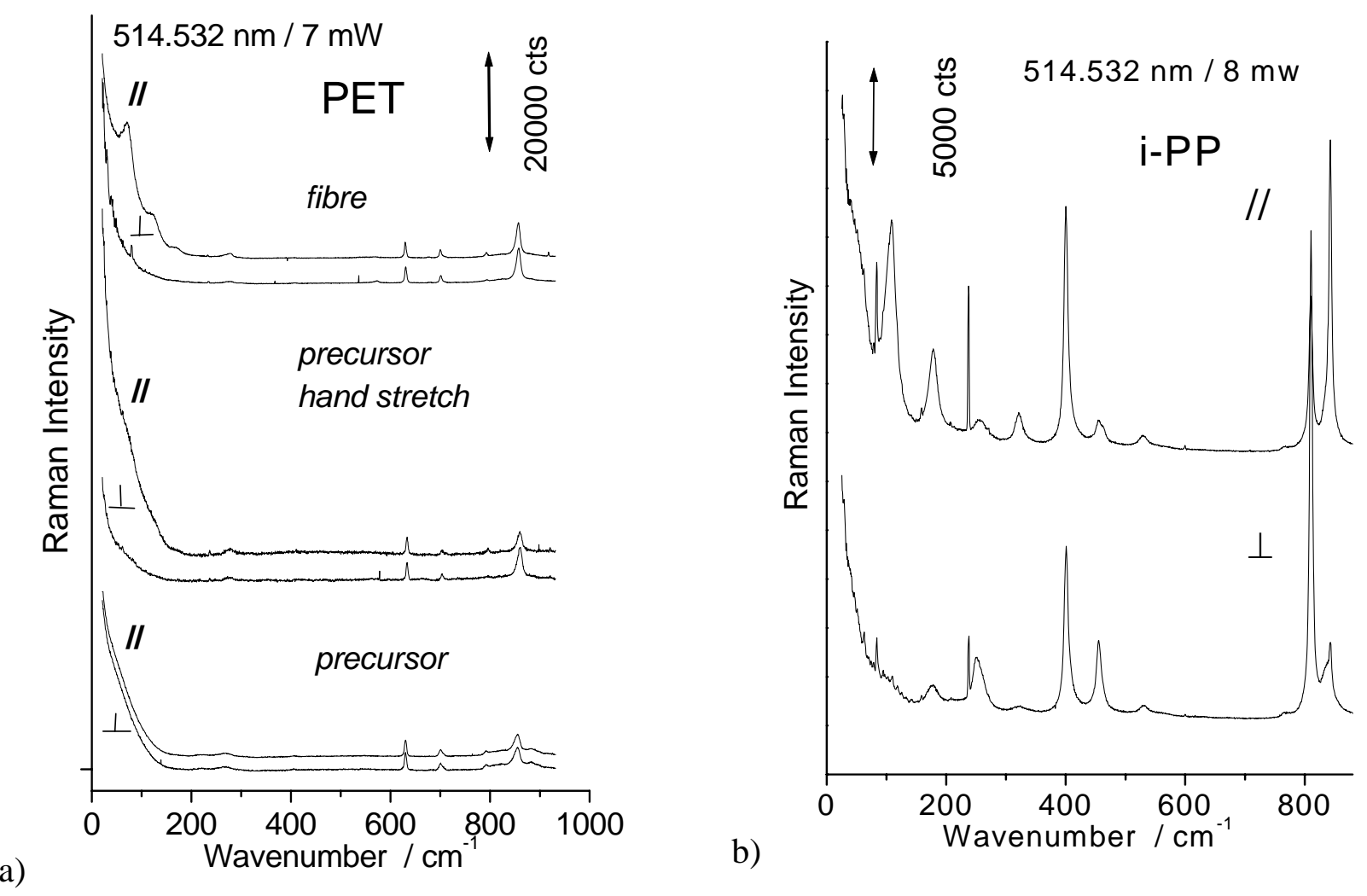

Fig. 3 : Polarised Raman spectra for a) pristine PET precursor (see micrograph in Fig. 2b), hand stretched precursor ( $\sim$ 10), standard PET fibre and $b)$ for $x 5$ stretched single I-PP fibre. 


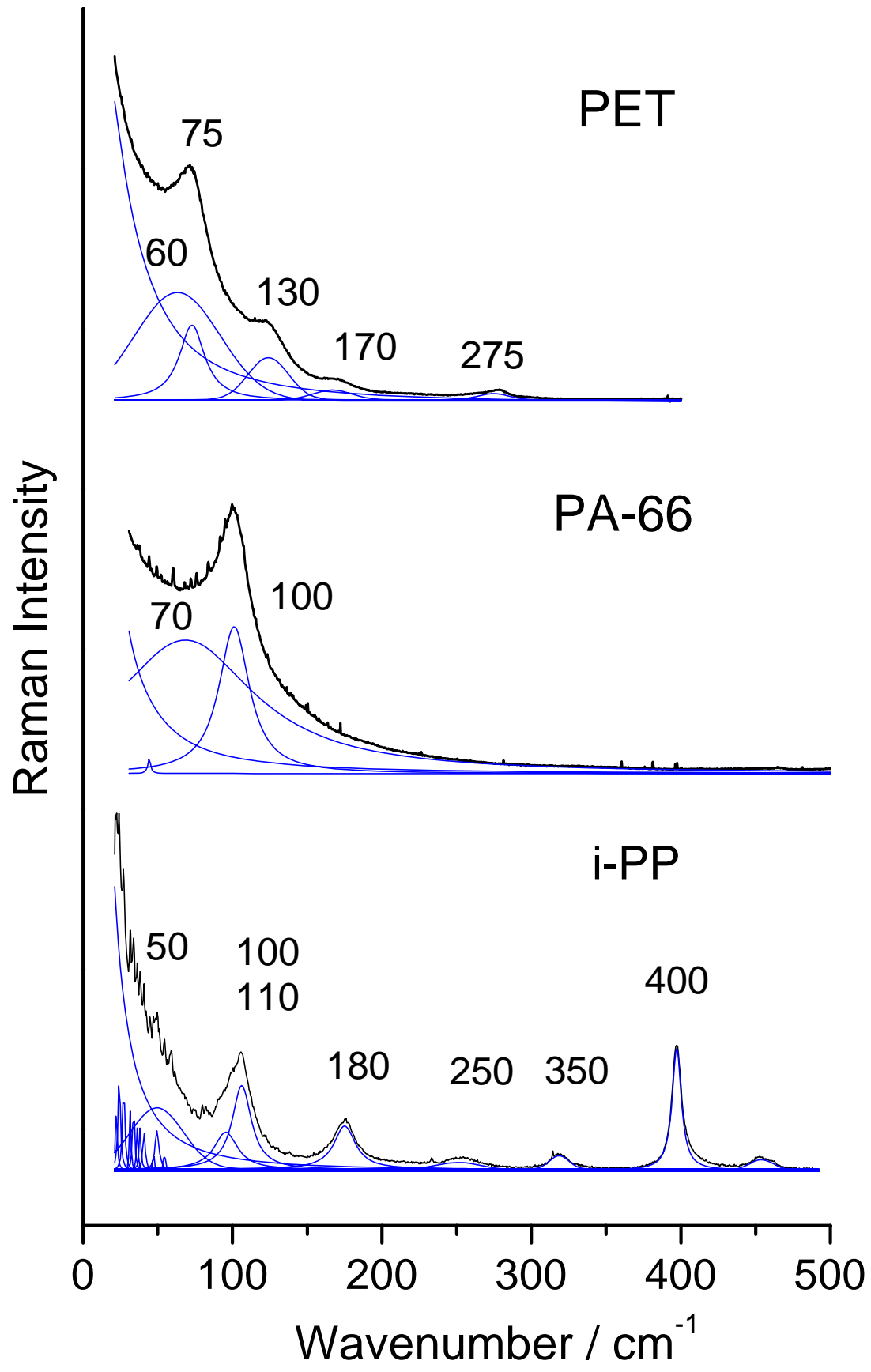

Fig. 4 : Low wavenumber parallel polarised Raman spectra for PET, PP and PA 66 fibres. Amorphous and crystalline chain components have been deduced. Note, the very narrow components are the Raman signature of air in microcracks and/or bubbles within the fibre. 

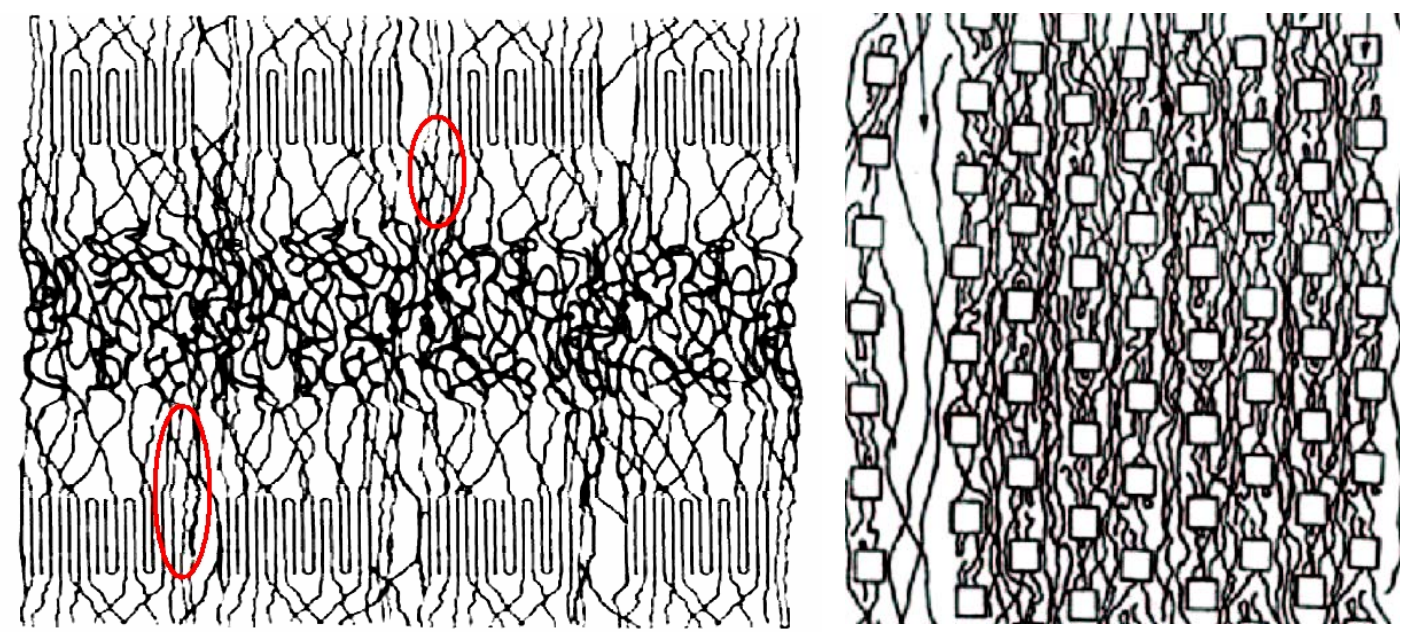

Fig. 5: Schematic view of the chain arrangements according to Prevorsek [4] and Oudet $[2,5]$ where crystalline and amorphous chains are more or less organised in series (paracrystal) or in parallel (ordered domains). Ordered parallel chains (including those in the circles often called oriented amorphous phase) will be considered as crystalline chains from the Raman point of view $[9,10]$. On the other hand, other chains will be considered as amorphous. 


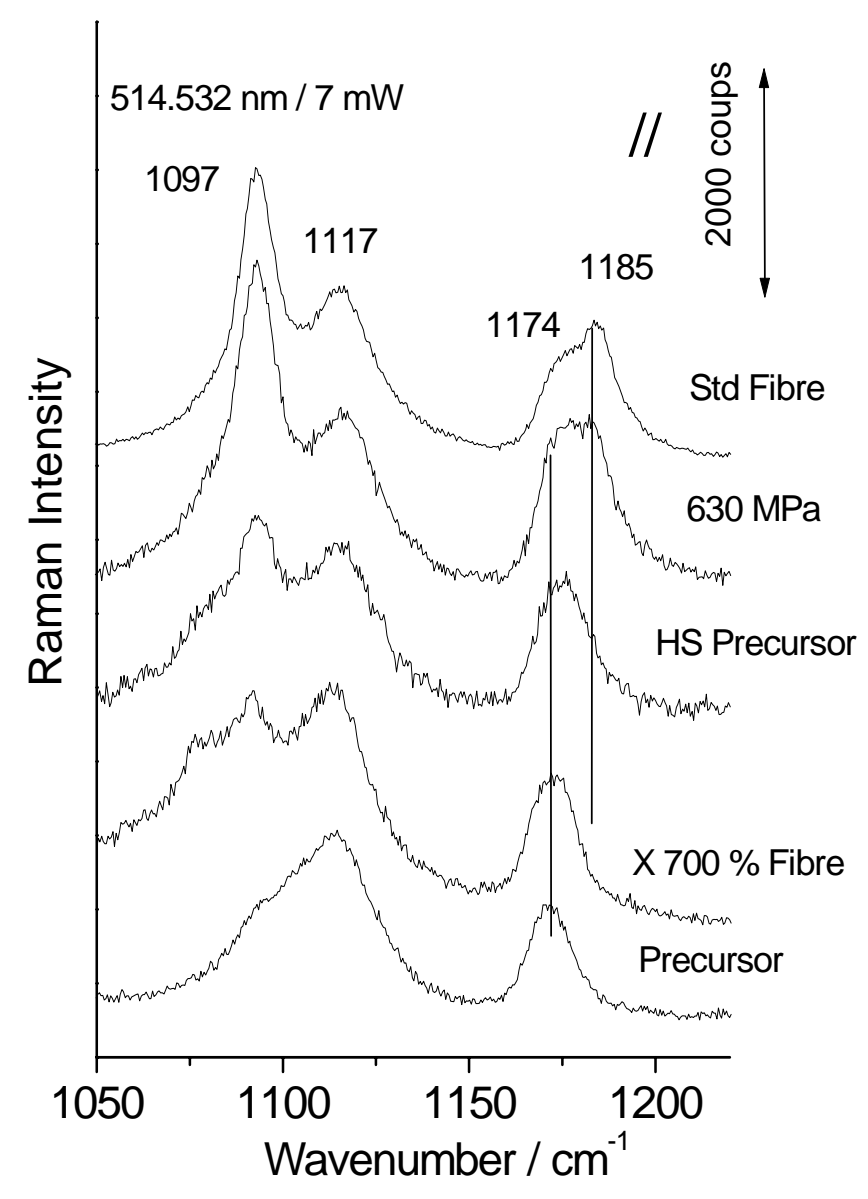

Fig. 6 : Comparison of parallel polarised Raman spectra recorded for more or less stretched PET precursor and for standard (Std) and $630 \mathrm{MPa}$ stretched fibre with the Universal Fibre Tester. HS : hand stretched ; $\mathrm{x} 700 \%$ : stretched $\mathrm{x} 7$ with the UFT. 
a)
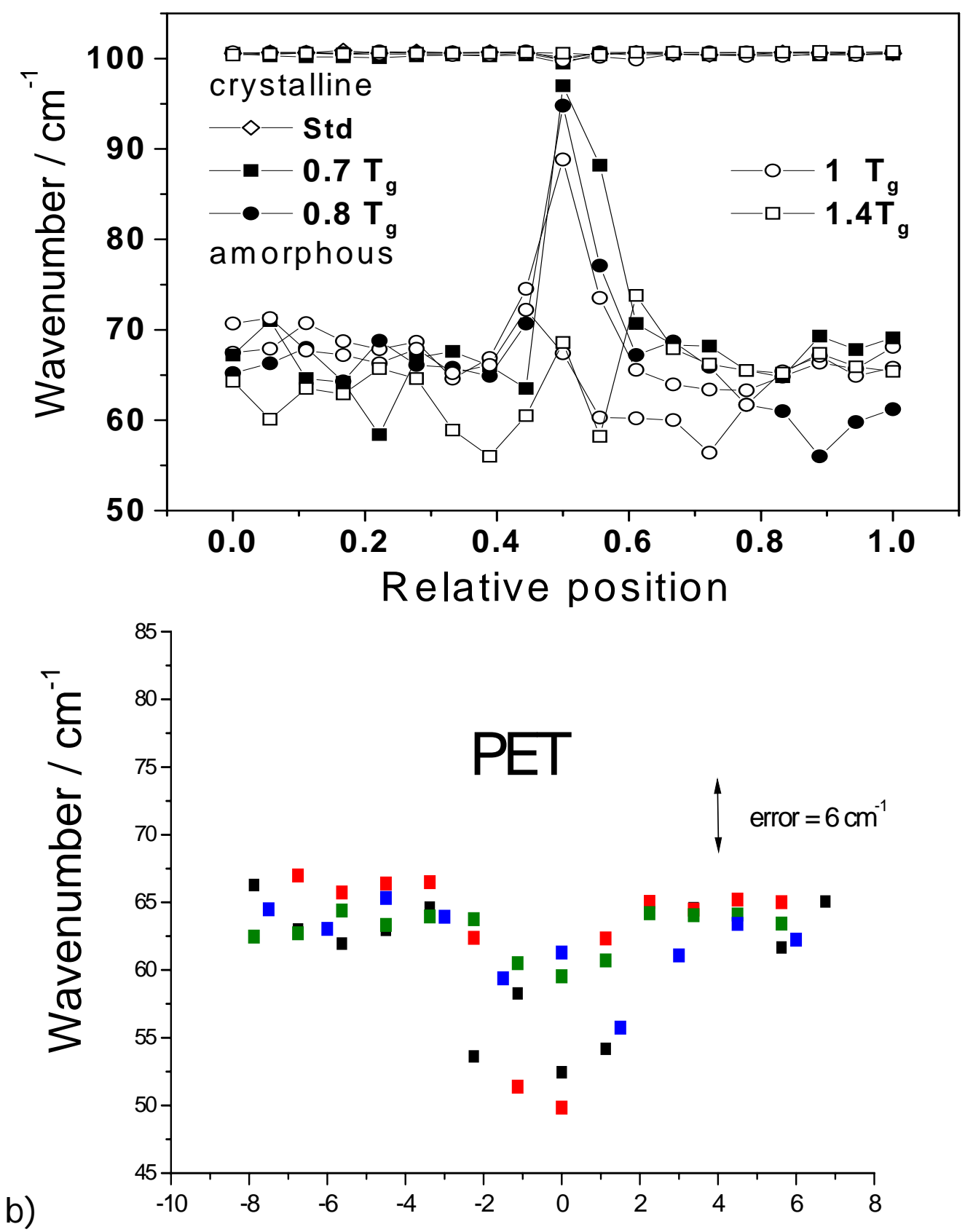

Fig. 7 : Plots of the crystalline (a), PA 66, 5 different fibres) and amorphous (a), PA 66 ; b) PET, 4 different fibres) component wavenumbers measured along line scans across fibre diameters for free standing fibres. PA 66 fibres are examined as-received (Std) and after different thermal treatments from 0.7 to $1.4 \mathrm{Tg}$ (after ref [11]). 


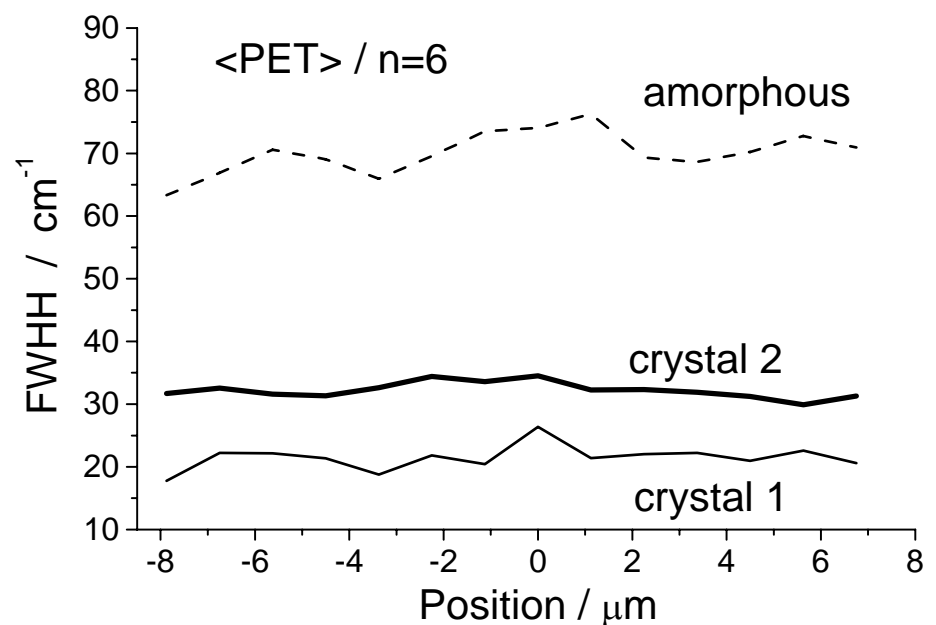

a)

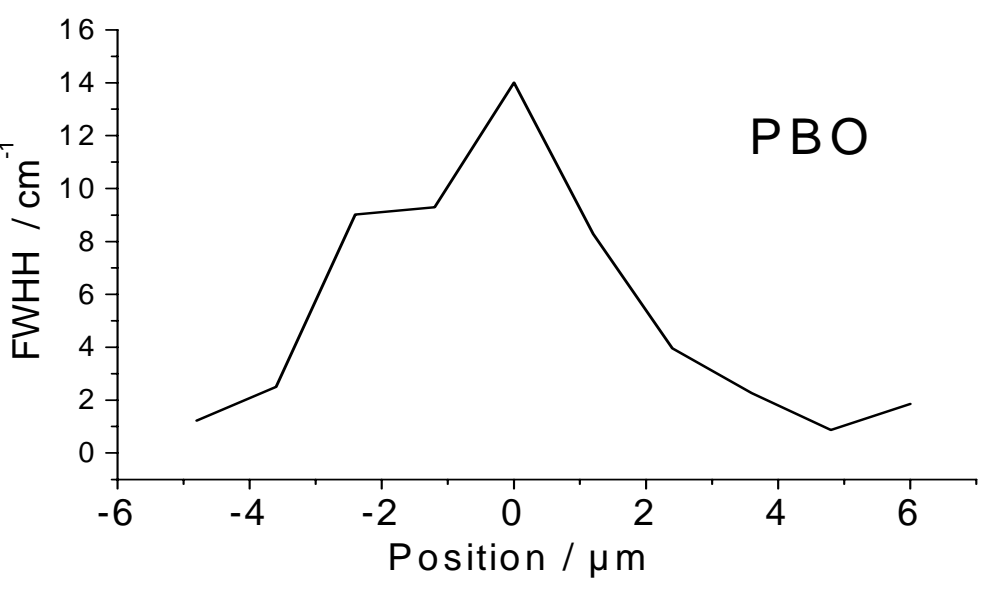

Fig. 8 : Plots of a) the PET (amorphous and 75 (crystal 1) and $130 \mathrm{~cm}^{-1}$ (crystal 2) components) and b) PBO (amorphous) component full width at half maximum (i.e. the sortrange disorder) measured along line scan across a fibre diameter for free standing fibres. 

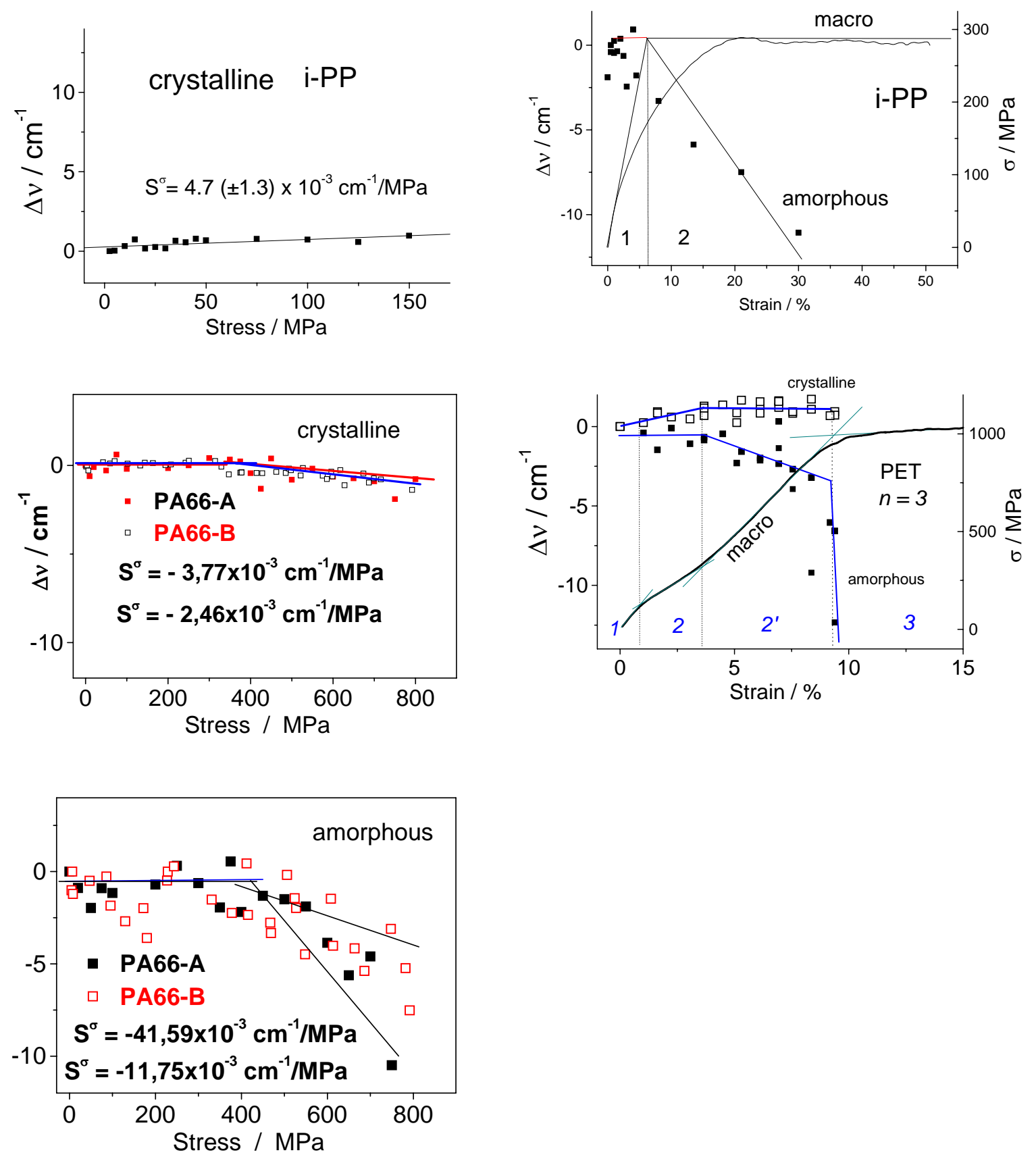

Fig. 9 : Wavenumber shift of the chain “ $\mathrm{T}$ ” “ mode (parallel polarisation) plotted as a function of increasing applied stress for i-PP and PA 66 fibres (strain for i-PP and PET). Crystalline and amorphous components are given and a comparison is made with macroscopic stress/strain behaviour for I-PP and PET fibres. Two series of PA 66 fibres from two different suppliers are analysed : black squares $=$ A fibre, open squares $=\mathrm{B}$ fibre . 


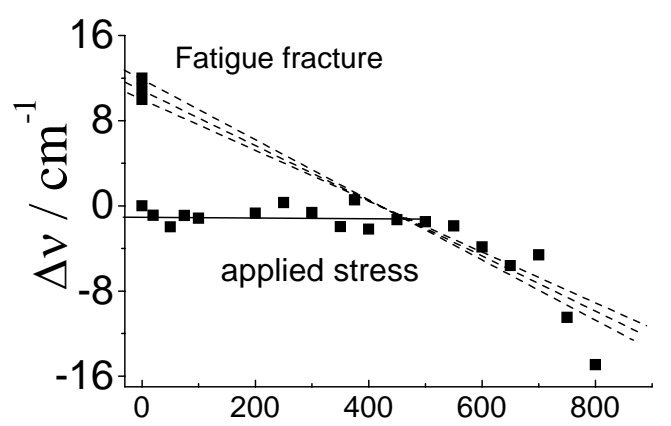

a)

Stress / MPa

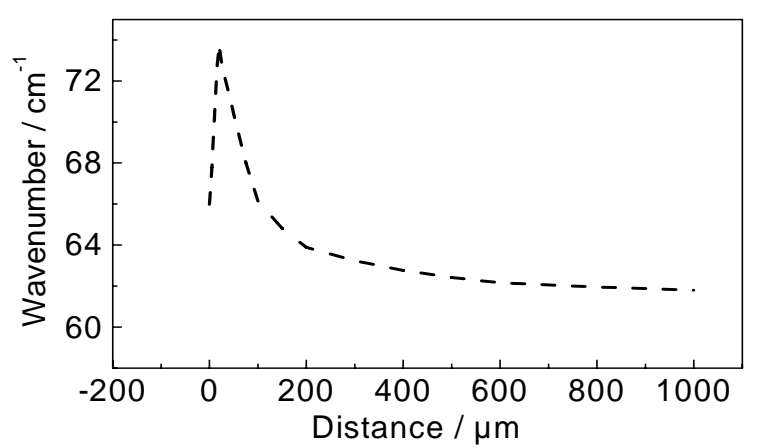

b)

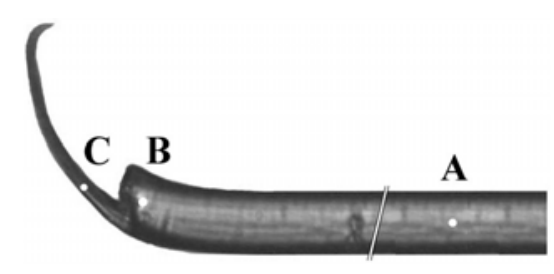

Fig. 10 : a) Comparison between the amorphous wavenumber shift measured as a function of the applied stress and the core maximum wavenumber shift measured at the fatigue fracture point for PA 66 fibre ; b) example of the amorphous maximum wavenumber of the " $T$ '” mode measured along line scan along the fibre axis from fracture B point (0 distance) to A point $(\sim 1000 \mu \mathrm{m})$ and symmetrically; fibre tested for $25 \times 10^{6}$ cycles (after [11]). 